BARI-TH/96-235

May 1996

\title{
On the Decay Mode $B^{-} \rightarrow \mu^{-} \bar{\nu}_{\mu} \gamma$
}

\author{
P. Colangelo ${ }^{a,}$ 中, F. De Fazio ${ }^{a, b}$, G. Nardulli ${ }^{a, b}$ 色 \\ ${ }^{a}$ Istituto Nazionale di Fisica Nucleare, Sezione di Bari, Italy \\ ${ }^{b}$ Dipartimento di Fisica, Universitá di Bari, Italy
}

\begin{abstract}
A QCD relativistic potential model is employed to compute the decay rate and the photon spectrum of the process $B^{-} \rightarrow \mu^{-} \bar{\nu}_{\mu} \gamma$. The result $\mathcal{B}\left(B^{-} \rightarrow\right.$ $\left.\mu^{-} \bar{\nu}_{\mu} \gamma\right) \simeq 1 \times 10^{-6}$ confirms the enhancement of this decay channel with respect to the purely leptonic mode, and supports the proposal of using this process to access relevant hadronic quantities such as the $B$-meson leptonic decay constant and the CKM matrix element $V_{u b}$.
\end{abstract}

\footnotetext{
${ }^{1}$ e-mail address: COLANGELO@BARI.INFN.IT

${ }^{2}$ e-mail address: NARDULLI@BARI.INFN.IT
} 
Noticeable theoretical attention has been recently given to the weak radiative decay

$$
B^{-} \rightarrow \mu^{-} \bar{\nu}_{\mu} \gamma
$$

The reason is in the peculiar role of this decay mode for the understanding of the dynamics of the annihilation processes occuring in heavy mesons [1, 2, 3, 4. Moreover, it has been observed that (11) can be studied to obtain indications on the value of the $B$-meson leptonic constant $f_{B}$ using a decay channel which differs from the purely leptonic modes $B^{-} \rightarrow \ell^{-} \bar{\nu}_{\ell}$, and is not hampered by the limitations affecting those latter processes. Such difficulties mainly consist in low decay rates ${ }^{3}$ (using $V_{u b}=3 \times 10^{-3}, f_{B}=200 \mathrm{MeV}$ and $\tau_{B^{-}}=1.646 \pm 0.063 \mathrm{ps}$ [5] one predicts $\mathcal{B}\left(B^{-} \rightarrow e^{-} \bar{\nu}_{e}\right) \simeq 6.610^{-12}$ and $\mathcal{B}\left(B^{-} \rightarrow \mu^{-} \bar{\nu}_{\mu}\right) \simeq$ $2.810^{-7}$ ) or in reconstruction problems for $B^{-} \rightarrow \tau^{-} \bar{\nu}_{\tau}$.

In ref. [4] heavy quark symmetry and experimental data on $D^{* 0} \rightarrow D^{0} \gamma$ have been exploited to study the dependence of $\mathcal{B}\left(B^{-} \rightarrow \mu^{-} \bar{\nu}_{\mu} \gamma\right)$ on the heavy meson decay constant $\hat{F} / \sqrt{m_{b}}$, which is the common value of $f_{B}$ and $f_{B^{*}}$ (modulo logarithmic factors) in the limit $m_{b} \rightarrow \infty$. The analysis is based on the dominance of polar diagrams contributing to the process $B^{-} \rightarrow \mu^{-} \bar{\nu}_{\mu} \gamma$, the pole being either the vector meson $B^{*}$ or the positive parity $J^{P}=1^{+}$state $B_{1}^{\prime}$ (see ref. [4] for further details). According to the analysis in [4], in correspondence to the expected range of values of $\hat{F}: \hat{F} \simeq 0.35 \mathrm{GeV}^{3 / 2}$, the branching ratio $\mathcal{B}\left(B^{-} \rightarrow \mu^{-} \bar{\nu}_{\mu} \gamma\right)$ should be $\mathcal{O}\left(10^{-6}\right)$, which represents an enhancement with respect to the purely leptonic mode.

In order to give further arguments in support of that analysis, we want to consider the process (11) in a different context. More precisely, whereas in [4] we have studied the feasibility of extracting $f_{B}=\hat{F} / \sqrt{m_{b}}$ from future experimental data, in this letter we study the decay (11) within a well defined theoretical model in order to have an independent estimate of the decay rate.

We employ a relativistic constituent quark model already used to study several aspects of the $B$-meson phenomenology [7, 8, 9]. Within this model the mesons are represented as bound states of valence quarks and antiquarks interacting via a QCD inspired istantaneous potential with a linear dependence at large distances, to account for confinement, and a modified coulombic behaviour at short distances to include the asymptotic freedom property of QCD. We adopt the interpolating form between such asymptotic dependences

\footnotetext{
${ }^{3}$ Present bounds are: $\mathcal{B}\left(B^{-} \rightarrow e^{-} \bar{\nu}_{e}\right)<1.510^{-5}, \mathcal{B}\left(B^{-} \rightarrow \mu^{-} \bar{\nu}_{\mu}\right)<2.110^{-5}$ [6].
} 
provided by the Richardson potential [10] In the rest frame, the state describing a $B_{a}$ meson is represented as:

$$
\left|B_{a}>=i \sum_{\alpha \beta} \frac{\delta_{\alpha \beta}}{\sqrt{3}} \sum_{r s} \frac{\delta_{r s}}{\sqrt{2}} \int d \vec{k}_{1} \psi_{B}\left(\vec{k}_{1}\right) b^{\dagger}\left(\vec{k}_{1}, r, \alpha\right) d_{a}^{\dagger}\left(-\vec{k}_{1}, s, \beta\right)\right| 0>,
$$

where $\alpha$ and $\beta$ are colour indices, $r$ and $s$ are spin indices, $b^{\dagger}$ and $d_{a}^{\dagger}$ are creation operators of the quark $b$ and the antiquark $\bar{q}_{a}$, carrying momenta $\vec{k}_{1}$ and $-\vec{k}_{1}$ respectively. The $B$ meson wave function $\psi_{B}\left(\vec{k}_{1}\right)$ satisfies a wave equation with relativistic kinematics (Salpeter equation) 11] taking the form (in the meson rest frame):

$$
\left\{\sqrt{\vec{k}_{1}^{2}+m_{b}^{2}}+\sqrt{\vec{k}_{1}^{2}+m_{q_{a}}^{2}}-M_{B_{a}}\right\} \psi_{B}\left(\vec{k}_{1}\right)+\int d \overrightarrow{k_{1}^{\prime}} V\left(\vec{k}_{1}, \vec{k}_{1}^{\prime}\right) \psi_{B}\left(\vec{k}_{1}^{\prime}\right)=0
$$

where $V\left(\vec{k}_{1}, \vec{k}_{1}^{\prime}\right)$ is the interaction potential in the momentum space and $\psi_{B}$ is covariantly normalized:

$$
\frac{1}{(2 \pi)^{3}} \int d \vec{k}_{1}\left|\psi_{B}\left(\vec{k}_{1}\right)\right|^{2}=2 M_{B_{a}}
$$

Solving eq.(3) by numerical methods one obtains the wave function; we shall present this result later on. It can be mentioned that by this model a number of predictions have been derived; for example, the heavy meson spectrum, leptonic constants [7, 8], semileptonic form factors and strong decay constants [9].

In the framework of the relativistic QCD potential model the wave function $\psi_{B}$ is the main dynamical quantity governing the decay (11). As a matter of fact, the amplitude of the process $B^{-}(p) \rightarrow \mu^{-}\left(p_{1}\right) \bar{\nu}_{\mu}\left(p_{2}\right) \gamma(k, \epsilon)$ can be written as

$$
\mathcal{A}\left(B^{-} \rightarrow \mu^{-} \bar{\nu}_{\mu} \gamma\right)=\frac{G_{F}}{\sqrt{2}} V_{u b}\left(L^{\mu} \cdot \Pi_{\mu}\right)
$$

where $G_{F}$ is the Fermi constant, $V_{u b}$ is the CKM matrix element involved in the decay, $L^{\mu}=\bar{\mu}\left(p_{1}\right) \gamma^{\mu}\left(1-\gamma_{5}\right) \nu\left(p_{2}\right)$ is the weak leptonic current, and $\Pi_{\mu}$ is defined by $\Pi_{\mu}=\Pi_{\mu \nu} \epsilon^{* \nu}$ ( $\epsilon$ is the photon polarization vector); $\Pi_{\mu \nu}$ represents the correlator

$$
\Pi_{\mu \nu}=i \int d^{4} x e^{i q \cdot x}<0\left|T\left[J_{\mu}(x) V_{\nu}(0)\right]\right| B(p)>
$$

In eq.(6) $q$ is $q=p_{1}+p_{2}, J_{\mu}(x)=\bar{u}(x) \gamma_{\mu}\left(1-\gamma_{5}\right) b(x)$ is the weak hadronic current and $V_{\nu}(0)=\frac{2}{3} e \bar{u}(0) \gamma_{\nu} u(0)-\frac{1}{3} e \bar{b}(0) \gamma_{\nu} b(0)$ is the electromagnetic (e.m.) current. The two

\footnotetext{
${ }^{4} \mathrm{~A}$ smearing of the Richardson potential at short distances has also been introduced to take into account the effects of the relativistic kinematics; see ref. [8] for the explicit form of the potential.
} 
pieces in the e.m. current correspond to the coupling to the light quark and to the heavy quark, respectively. The corresponding contributions to $\Pi_{\mu \nu}$ will be referred to as $\Pi_{\mu \nu}^{\ell}$ and $\Pi_{\mu \nu}^{h}$, depicted in figs. 1a,b. $\Pi_{\mu \nu}^{\ell}$ contains the light quark propagator:

$$
S_{u}(x, 0)=\int \frac{d^{4} \ell}{(2 \pi)^{4}} \frac{e^{i \ell \cdot x}}{\ell^{2}-m_{u}^{2}}\left(\ell+m_{u}\right)
$$

while $\Pi_{\mu \nu}^{h}$ contains the analogous $b$ quark propagator.

The calculation of the time-ordered product appearing in (6) gives:

$$
\Pi_{\mu \nu}^{\ell}=-\frac{2}{3} e \int d^{4} x e^{i q \cdot x} \int \frac{d^{4} \ell}{(2 \pi)^{4}} \frac{e^{i \ell \cdot x}}{\ell^{2}-m_{u}^{2}}<0\left|\tilde{J}_{\mu \nu}^{\ell}\right| B>.
$$

The analogous expression for $\Pi_{\mu}^{h}$ can be obtained by the replacements: $\frac{2}{3} \leftrightarrow-\frac{1}{3}, m_{u} \leftrightarrow m_{b}$, $\tilde{J}_{\mu \nu}^{\ell} \leftrightarrow \tilde{J}_{\mu \nu}^{h}$. The operators $\tilde{J}_{\mu \nu}^{\ell}$ and $\tilde{J}_{\mu \nu}^{h}$, which depend on the integration variable $x$, are written as $\tilde{J}_{\mu \nu}^{\ell}=\bar{u}(0) \Gamma_{\mu \nu}^{\ell} b(x)$ and $\tilde{J}_{\mu \nu}^{h}=\bar{u}(x) \Gamma_{\mu \nu}^{h} b(0)$, with the $\Gamma$ matrices given by $\Gamma_{\mu \nu}^{\ell}=\gamma_{\nu}\left(\ell+m_{u}\right) \gamma_{\mu}\left(1-\gamma_{5}\right)$ and $\Gamma_{\mu \nu}^{h}=\gamma_{\mu}\left(1-\gamma_{5}\right)\left(\ell+m_{b}\right) \gamma_{\nu}$. In the constituent quark model such operators can be expressed in terms of quark operators; for example one has:

$$
\begin{aligned}
\tilde{J}_{\mu \nu}^{\ell}= & \sum_{\alpha \beta} \sum_{r s} \delta_{\alpha \beta} \int \frac{d^{3} q_{1} d^{3} q_{2}}{(2 \pi)^{3}}\left[\frac{m_{b} m_{u}}{E_{u}\left(\vec{q}_{1}\right) E_{b}\left(\vec{q}_{2}\right)}\right]^{1 / 2} \\
: & {\left[\bar{u}_{u}\left(\vec{q}_{1}, r\right) b_{u}^{\dagger}\left(\vec{q}_{1}, r, \alpha\right)+\bar{v}_{u}\left(\vec{q}_{1}, r\right) d_{u}\left(\vec{q}_{1}, r, \alpha\right)\right] \Gamma_{\mu \nu}^{\ell} } \\
& {\left[u_{b}\left(\vec{q}_{2}, s\right) b_{b}\left(\vec{q}_{2}, s, \beta\right) e^{-i q_{2} \cdot x}+v_{b}\left(\vec{q}_{2}, s\right) d_{b}^{\dagger}\left(\vec{q}_{2}, s, \beta\right) e^{i q_{2} \cdot x}\right]: }
\end{aligned}
$$

where $E_{q}(\vec{q})=\sqrt{\vec{q}^{2}+m_{q}^{2}}$ and $u_{q}\left(v_{q}\right)$ are quark (antiquark) spinors.

By exploiting anticommutation relations among annihilation and creation operators, we obtain, in the $B$ meson rest frame:

$$
\begin{aligned}
& \Pi_{\mu \nu}=i \frac{e}{\sqrt{6}} \sum_{r} \int \frac{d^{3} k_{1}}{(2 \pi)^{3}} \psi_{B}\left(\vec{k}_{1}\right)\left[\frac{m_{b} m_{u}}{E_{b}\left(\vec{k}_{1}\right) E_{u}\left(\vec{k}_{1}\right)}\right]^{1 / 2} \\
& \bar{v}_{u}\left(-\vec{k}_{1}, r\right)\left\{-2 \frac{\gamma_{\nu}\left[\left(\not q_{2}-\not 1\right)+m_{u}\right] \gamma_{\mu}\left(1-\gamma_{5}\right)}{\left(q_{2}-q\right)^{2}-m_{u}^{2}}+\frac{\gamma_{\mu}\left(1-\gamma_{5}\right)\left[\left(\not 1-\not q_{1}\right)+m_{b}\right] \gamma_{\nu}}{\left(q_{1}-q\right)^{2}-m_{b}^{2}}\right\} u_{b}\left(\vec{k}_{1}, r\right)
\end{aligned}
$$

where $q_{1}=\left(E_{u},-\vec{k}_{1}\right)$ and $q_{2}=\left(E_{b}, \vec{k}_{1}\right)$. We recognize in the two factors in the curly brakets the contributions of $\Pi_{\mu \nu}^{\ell}$ and $\Pi_{\mu \nu}^{h}$, respectively.

Since $\Pi_{\mu \nu}$ only depends on two vectors, the $B$ meson momentum $p_{\mu}$ and the photon momentum $k_{\mu}$, it can be written in terms of six independent Lorentz structures:

$$
\Pi_{\mu \nu}=\alpha p_{\mu} p_{\nu}+\beta k_{\mu} k_{\nu}+\zeta k_{\mu} p_{\nu}+\delta p_{\mu} k_{\nu}+\xi g_{\mu \nu}+i \eta \epsilon_{\mu \nu \rho \sigma} p^{\rho} k^{\sigma}
$$


By gauge invariance one has $\alpha=0$ and $\xi=-p \cdot k \zeta$; moreover, after saturation by $\epsilon^{* \nu}$ one gets:

$$
\Pi_{\mu}=\Pi_{\mu \nu} \epsilon^{* \nu}=\left[\zeta\left(k_{\mu} p_{\nu}-p \cdot k g_{\mu \nu}\right)+i \eta \epsilon_{\mu \nu \rho \sigma} p^{\rho} k^{\sigma}\right] \epsilon^{* \nu}
$$

i.e. only the terms proportional to $\eta$ and $\zeta$ survive: they are the vector and the axial vector contribution, respectively. It is convenient to compute eq.(12) in the $B$ rest frame $p=\left(M_{B}, \overrightarrow{0}\right)$, with $k=\left(k^{0}, 0,0, k^{0}\right)$; the result reads:

$$
\zeta=\frac{\Pi_{11}}{M_{B} k^{0}} \quad \eta=\frac{\Pi_{12}}{i M_{B} k^{0}} .
$$

At this point, it is straightforward to calculate the rate of the decay process (1); one obtains $\left(m_{\mu} \simeq 0\right)$ :

$$
\Gamma\left(B^{-} \rightarrow \mu^{-} \bar{\nu}_{\mu} \gamma\right)=\frac{G_{F}^{2}\left|V_{u b}\right|^{2}}{3(2 \pi)^{3}} \int_{0}^{M_{B} / 2} d k^{0} k^{0}\left(M_{B}-2 k^{0}\right)\left[\left|\Pi_{11}\right|^{2}+\left|\Pi_{12}\right|^{2}\right]
$$

where:

$$
\begin{aligned}
\Pi_{11} & =\frac{i e}{4 \sqrt{3} \pi} \int_{-1}^{1} d \cos \theta \int_{0}^{\left|\vec{k}_{1}\right|_{\max }}\left|\vec{k}_{1}\right| d\left|\vec{k}_{1}\right| u_{B}\left(\left|\vec{k}_{1}\right|\right)\left[\frac{1}{E_{b} E_{u}\left(E_{b}+m_{b}\right)\left(E_{u}+m_{u}\right)}\right]^{1 / 2} \\
\{ & -\frac{2}{f}\left[\left(M_{B}-k^{0}\right)\left[\left(E_{b}+m_{b}\right)\left(E_{u}+m_{u}\right)-\left|\vec{k}_{1}\right|^{2}\right]+\left|\vec{k}_{1}\right|^{2} \cos ^{2} \theta\left(E_{u}+m_{u}-E_{b}-m_{b}\right)\right. \\
& +\left|\vec{k}_{1}\right| k^{0} \cos \theta\left(E_{u}+m_{u}-E_{b}-m_{b}\right) \\
& \left.-\left(E_{b}+m_{b}\right)\left(E_{u}+m_{u}\right)\left(E_{b}+m_{u}\right)+\left(E_{b}-m_{u}\right)\left|\vec{k}_{1}\right|^{2}\right] \\
& +\frac{1}{g}\left[\left(M_{B}-k^{0}\right)\left[\left|\vec{k}_{1}\right|^{2}-\left(E_{b}+m_{b}\right)\left(E_{u}+m_{u}\right)\right]+\left|\vec{k}_{1}\right|^{2} \cos ^{2} \theta\left(E_{u}+m_{u}-E_{b}-m_{b}\right)\right. \\
& -\left|\vec{k}_{1}\right| k^{0} \cos \theta\left(E_{u}+m_{u}-E_{b}-m_{b}\right) \\
& \left.\left.+\left(E_{b}+m_{b}\right)\left(E_{u}+m_{u}\right)\left(E_{u}+m_{b}\right)-\left(E_{u}-m_{b}\right)\left|\vec{k}_{1}\right|^{2}\right]\right\}
\end{aligned}
$$

and

$$
\begin{aligned}
\Pi_{12}= & \frac{e}{4 \sqrt{3} \pi} \int_{-1}^{1} d \cos \theta \int_{0}^{\left|\vec{k}_{1}\right|_{\max }}\left|\vec{k}_{1}\right| d\left|\vec{k}_{1}\right| u_{B}\left(\left|\vec{k}_{1}\right|\right)\left[\frac{1}{E_{b} E_{u}\left(E_{b}+m_{b}\right)\left(E_{u}+m_{u}\right)}\right]^{1 / 2} \\
& k^{0}\left[\left(E_{b}+m_{b}\right)\left(E_{u}+m_{u}\right)-\left|\vec{k}_{1}\right|^{2}\right]+\left|\vec{k}_{1}\right| \cos \theta\left(m_{b}-m_{u}\right)\left(E_{u}+m_{u}+E_{b}+m_{b}\right) \\
+ & \left.\left|\vec{k}_{1}\right| \cos \theta\left(M_{B}-k^{0}\right)\left(E_{u}+m_{u}-E_{b}-m_{b}\right)\right\}\left(\frac{2}{f}-\frac{1}{g}\right) .
\end{aligned}
$$

In eqs.(15,16) the quantities $f$ and $g$ are defined as

$$
f=m_{b}^{2}+M_{B}^{2}-2 M_{B} k^{0}-2 M_{B} E_{b}+2 E_{b} k^{0}-2 k^{0}\left|\vec{k}_{1}\right| \cos \theta-m_{u}^{2}
$$




$$
g=m_{u}^{2}+M_{B}^{2}-2 M_{B} k^{0}-2 M_{B} E_{u}+2 E_{u} k^{0}+2 k^{0}\left|\vec{k}_{1}\right| \cos \theta-m_{b}^{2},
$$

whereas the S-wave reduced function $u$ is related to $\psi_{B}$ :

$$
u_{B}\left(\left|\vec{k}_{1}\right|\right)=\frac{\left|\vec{k}_{1}\right|}{\sqrt{2} \pi} \psi_{B}\left(\vec{k}_{1}\right) ;
$$

we plot in fig.2 the function $u_{B}$ obtained as a solution of the wave equation (3).

Let us now point out that in computing the diagrams in figs.1a,b and, therefore, in eqs.(15), (16), we have so far imposed 4-momentum conservation for the physical particles $B, \mu, \nu$ and $\gamma$ in the process (11). On the other hand, energy conservation has to be imposed also at quark level since, otherwise, (15) and (16) would present spurious kinematical singularities. In order to deal with this problem we follow the approach originally proposed within the ACCMM model [12] for the decay $b \rightarrow u \ell \bar{\nu}_{\ell}$. One assumes that the spectator quark has a definite mass, while the active quark has a "running" mass, defined consistently with the energy conservation:

$$
E_{b}+E_{u}=M_{B}
$$

Therefore, as in the case of the ACCMM model, the running mass of the active $b$ quark can be defined by:

$$
m_{b}^{2}\left(\vec{k}_{1}\right)=M_{B}^{2}+m_{u}^{2}-2 M_{B} \sqrt{\vec{k}_{1}^{2}+m_{u}^{2}} .
$$

Moreover, by requiring that the right hand side of eq. (21) is positive, an upper bound on the quark momentum $\left|\vec{k}_{1}\right|$ : can be obtained

$$
\left|\vec{k}_{1}\right| \leq \frac{M_{B}^{2}-m_{u}^{2}}{2 M_{B}} .
$$

Notice that the masses of the light constituent quarks, as obtained by fits to the meson spectrum, are $m_{u}=m_{d}=38 \mathrm{MeV}$.

The contribution of the two physical processes when the photon couples to the light or to the heavy quark is still recognizable in eqs. (15)-(16), since the quantities $f$ and $g$ come from the light and heavy quark propagators respectively. It turns out that the contribution of the terms proportional to $\frac{1}{g}$ are numerically much smaller than those proportional to $\frac{1}{f}$. This is not surprising, since the e.m. coupling of the photon to the quarks corresponds to a magnetic transition, and therefore it is inversely proportional to the quark mass. 
A final remark concerns the photon energy. In eq.(14) we have allowed $k^{0}$ to vary in the range $\left[0, M_{B} / 2\right]$; however the integral diverges at the lower limit $k^{0}=0$. This result is unphysical since it would correspond to a zero energy photon in the final state. Formally, this divergence would be canceled by radiative corrections to the formulae (15) and (16). On the other hand, one should take into account that at future experiments, e.g. at the SLAC $B$-factory, the smallest measurable photon energy is of the order of $50 \mathrm{MeV}$; therefore, it is a reasonable assumption to cut off from the integral the small photon energies, and, at the same time, to neglect radiative corrections.

In our calculation, the effect of the unphysical divergence begins around a photon energy $k^{0} \simeq 350 \mathrm{MeV}$, and therefore we use this value as a lower bound for the photon energy.

In fig. 3 we plot the photon spectrum for the decay (目); the differential distribution has a peak around $1.5 \mathrm{GeV}$, which should render it quite accessible to experimental analyses.

For the decay width we obtain the result $\Gamma\left(B^{-} \rightarrow \mu^{-} \bar{\nu}_{\mu} \gamma\right)=3.710^{-19}\left(\frac{V_{u b}}{3 \times 10^{-3}}\right)^{2} \mathrm{GeV}$, which corresponds to

$$
\mathcal{B}\left(B^{-} \rightarrow \mu^{-} \bar{\nu}_{\mu} \gamma\right)=0.910^{-6}
$$

This result is obtained with a cut-off $\Delta=350 \mathrm{MeV}$ in the photon energy. As it can be seen from fig.3, the uncertainty related to this choice should not be significant. For example, putting $\Delta=100 \mathrm{MeV}$ the result for the branching ratio increases by less than $10 \%$.

The conclusion we can draw from this result is that the relativistic quark model gives predictions in agreement with the expectations discussed in ref. [4]; the weak radiative decay $B^{-} \rightarrow \mu^{-} \bar{\nu}_{\mu} \gamma$ has an appreciable rate and might be observed in the near future.

As for on the theoretical uncertainties of the result (23), a part from the energy cut-off they mainly come from the choice of the $B$ meson wave function. A possible estimate of this theoretical error consists in assuming a different wave function. A commonly used quark momentum distribution inside the $B$ meson is given by the ACCMM model [12]; in our notations it corresponds to the gaussian wave function:

$$
u_{B}\left(\vec{k}_{1}\right)=2\left|\vec{k}_{1}\right|\left(\frac{2 M_{B}}{\sqrt{\pi} P_{F}^{3}}\right)^{1 / 2} \exp \left(-\frac{\vec{k}_{1}^{2}}{2 P_{F}^{2}}\right) .
$$

The parameter $P_{F}$ is related to the heavy quark average square momentum: $\left\langle p^{2}\right\rangle=\frac{3}{2} P_{F}^{2}$. In a recent analysis 13 this parameter has been fitted using experimental data for the 
inclusive decay $B \rightarrow X_{c} \ell \bar{\nu}_{\ell}$ [14 with the result: $P_{F}=0.51 \mathrm{GeV}$. Using (24) with such a value of $P_{F}$ in the previous formulas, one would obtain $\mathcal{B}\left(B^{-} \rightarrow \mu^{-} \bar{\nu}_{\mu} \gamma\right) \simeq 0.8 \cdot 10^{-6}$. This result suggests that the estimate given in eq. (23) is rather accurate; the rate for the weak radiative decay $B^{-} \rightarrow \mu^{-} \bar{\nu}_{\mu} \gamma$ is large enough for a measurement at future accelerators.

Acknowledgements

We thank M.Carpinelli, M. Giorgi, A. Palano and N.Paver for interesting discussions. 


\section{References}

[1] G. Burdman, T. Goldman and D. Wyler, Phys. Rev. D 51 (1995) 111.

[2] D. Atwood, G. Eilam and A. Soni, preprint TECHNION-PH-94-13, hep-ph/9411367.

[3] G.Eilam, I. Halperin and R.R.Mendel, Phys. Lett. B 361 (1995) 137.

[4] P. Colangelo, F. De Fazio and G. Nardulli, Phys. Lett. B 372 (1996) 331.

[5] T. E. Browder and K. Honscheid, Prog. Part. Nucl. Phys. 35 (1995) 81.

[6] CLEO Collaboration, M. Artuso et al., Phys. Rev. Lett. 75 (1995) 785.

[7] P. Cea, P. Colangelo, L. Cosmai and G. Nardulli, Phys. Lett. B 206 (1988) 691.

[8] P. Colangelo, G. Nardulli and M. Pietroni, Phys. Rev. D 43 (1991) 3002.

[9] P. Colangelo, G. Nardulli and L.Tedesco, Phys. Lett. B 271 (1991) 344;

P. Colangelo, F. De Fazio and G. Nardulli, Phys. Lett. B 334 (1994) 175.

[10] J. L. Richardson, Phys. Lett. B 82 (1979) 272.

[11] E. E. Salpeter, Phys. Rev. 87 (1952) 328.

[12] G. Altarelli, N. Cabibbo, G. Corbó, L. Maiani and G. Martinelli, Nucl. Phys. B 208 (1982) 365 .

[13] D. S. Hwang, C. S. Kim and W. Namgung, preprint KEK-TH-473, hep-ph/9604225.

[14] CLEO Collaboration, B. Barish et al., Phys. Rev. Lett. 76 (1996) 1570. 


\section{Figure Captions}

Figure 1

Diagrams describing the decay $B^{-} \rightarrow \mu^{-} \bar{\nu}_{\mu} \gamma$.

Figure 2

The wave function $u_{B}\left(k_{1}\right)$ as obtained by the QCD relativistic quark model.

Figure 3

Predicted photon energy spectrum. 


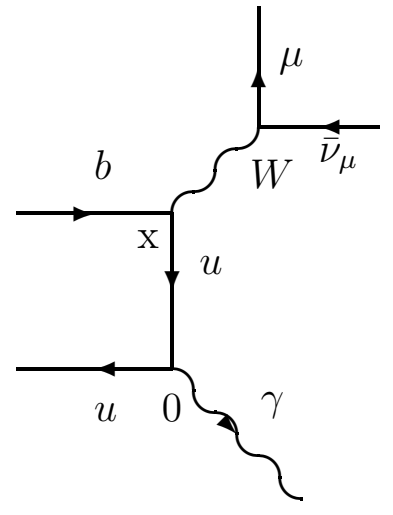

Fig. 1 a

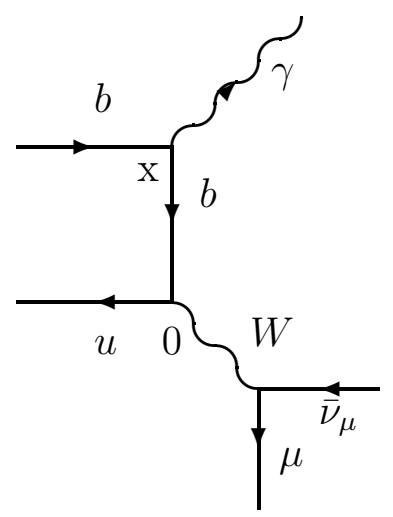

Fig. 1 b 


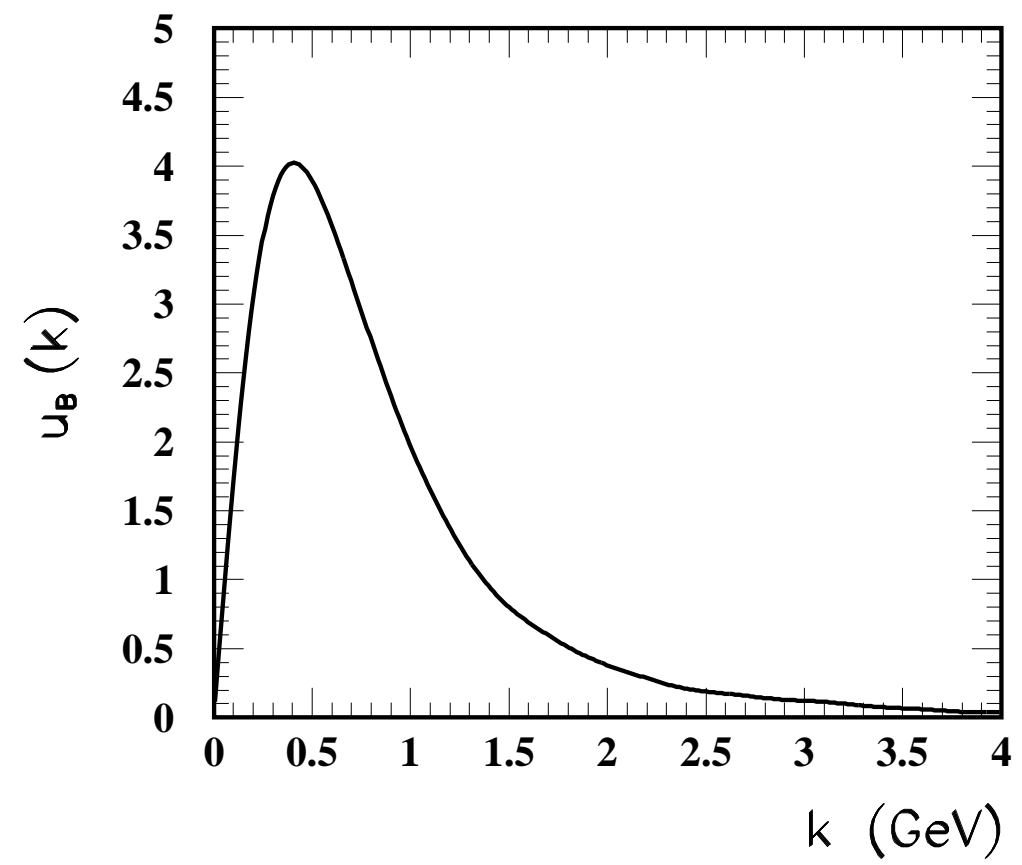

fig. 2 


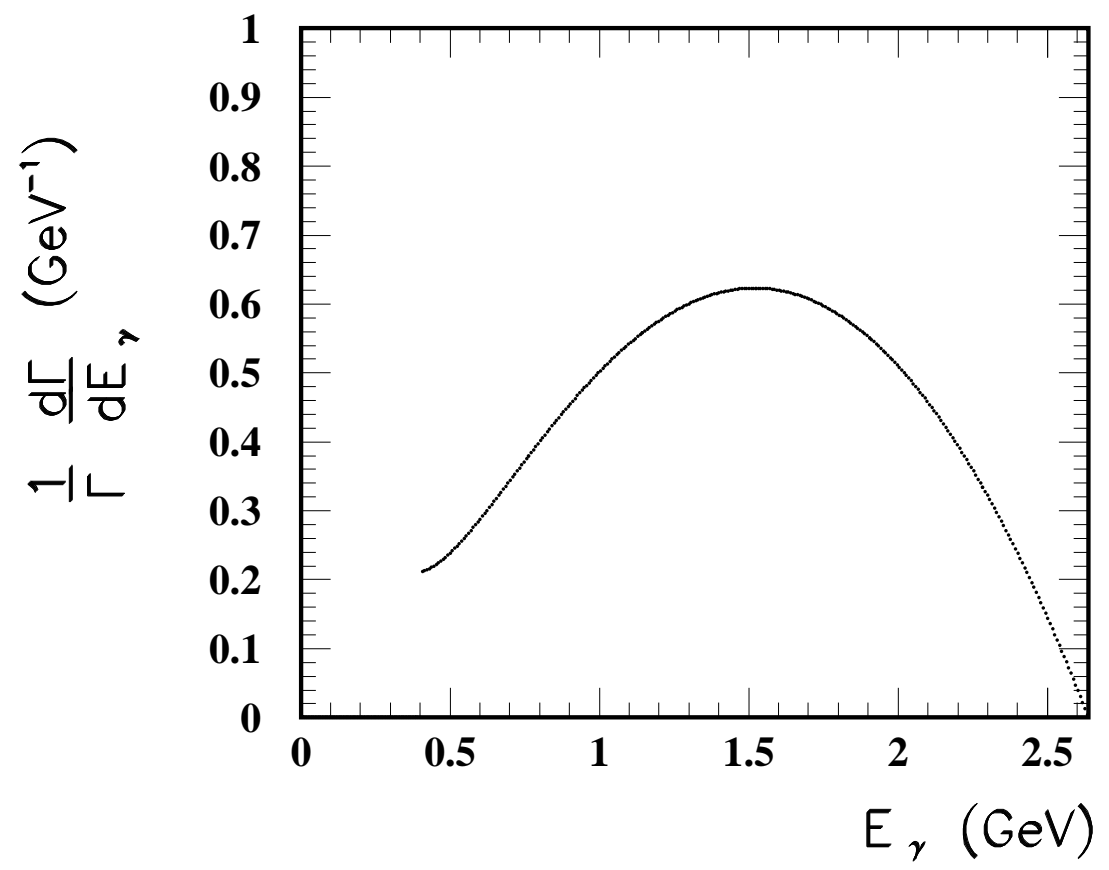

fig. 3 